\title{
Editorial. Mort volontaire combattante : sacrifices et stratégies
}

\section{(2) OpenEdition \\ Journals}

Édition électronique

URL : http://journals.openedition.org/conflits/2086

DOI : $10.4000 /$ conflits.2086

ISSN : $1777-5345$

Éditeur :

CCLS - Centre d'études sur les conflits lilberté et sécurité, L'Harmattan

Édition imprimée

Date de publication : 1 novembre 2006

Pagination : 7-9

ISBN : 2-296-01436-4

ISSN : 1157-996X

Référence électronique

" Editorial. Mort volontaire combattante : sacrifices et stratégies », Cultures \& Conflits [En ligne], 63 automne 2006, mis en ligne le 09 novembre 2006, consulté le 30 mars 2021. URL : http:// journals.openedition.org/conflits/2086; DOI : https://doi.org/10.4000/conflits.2086

Ce document a été généré automatiquement le 30 mars 2021.

Creative Commons License 


\section{Editorial. Mort volontaire combattante : sacrifices et stratégies}

«Il se pencha fortement, et la maison tomba sur les princes et sur tout le peuple qui y était. Ceux qu'il fit périr à sa mort furent plus nombreux que ceux qu'il avait tués pendant sa vie ${ }^{1} »$.

1 Le présent numéro qui traite de la «mort volontaire combattante » met l'accent sur le contexte polémique de ce geste et indique fortement l'intention stratégique de ses auteurs ou promoteurs, sans que pour autant nous ne nous fassions beaucoup d'illusions sur la dimension rationnelle et l'efficacité de ces calculs ${ }^{2}$. Il souligne également la dynamique de l'acte et la complexité des motivations qui y mènent, ce qui exclut de nos analyses les explications purement individuelles - psychologiques et bien sûr psychiatriques -; nous utilisons le terme de "sacrifice» pour qualifier cette démarche. Nous n'avons pas repris dans notre titre le mot "kamikaze", même si l'actualité en recommande l'usage, parce qu'il pose problème.

2 L'expression, on le sait, fait référence à un vent divin qui avait stoppé au xiiie siècle, l'invasion mongole du Japon en dispersant opportunément la flotte d'invasion mongole. Le nom a été réutilisé pour désigner les missions suicides d'aviateurs japonais pendant la Seconde Guerre mondiale ${ }^{3}$; mais, parallèlement, les Américains l'ont interprété dans le sens de folie fanatique et c'est avec cette deuxième acception qu'il est entré dans le vocabulaire international. Le réemploi du terme dans les médias - depuis 2001 renvoie aux mêmes ambiguïtés : fanatisme pour les uns, sacrifice pour les autres, le tout dans un contexte où les dimensions religieuses, politiques et militaires se mêlent inextricablement. Notre page de couverture l'atteste, le « juge » Samson est un modèle d'un genre qui s'inscrit dans la longue durée.

La première nécessité était de clarifier une notion fort complexe dont les usages obscurcissent de surcroît le contenu. Pour ce faire, nous sommes partis de la dimension polémique du terme, de son oscillation entre légitimité et illégitimité, qui, non seulement oppose principalement l'Occident à un certain islam, mais qui, comme le rappelle opportunément Bruno Etienne, traverse le monde musulman lui-même. Si dans nos esprits occidentaux le kamikazat est indissociable du fanatisme - japonais hier, musulman aujourd'hui - ailleurs, une partie de la presse ou de l'opinion célèbre les bombes humaines comme des martyrs! Martyrs, c'est-à-dire témoins, exemples 
donc à imiter et avec d'autant moins de retenue que, comme nous dit le discours justificatif de victimisation, l'extrême violence du kamikazat n'est que la transformation de la pression insupportable que subissent des individus occupés, aliénés, dépossédés.

L'analyse de la "réception » du phénomène, que mènent Daniel Hermant, en ce qui concerne le 11 septembre et ses suites en Occident et Laetitia Bucaille pour le kamikazat lié à l'Intifada al-Aqsa, montre comment se développe une véritable topique de l'indignation qui pousse les sociétés cibles à l'hyper-réaction. Cette situation hypothèque également les mentalités par l'angoisse qu'elle sécrète : angoisse du chaos et du déséquilibre - qui n'est pas simple pusillanimité de gens trop bien nourris - en Occident, angoisse devant les menaces de destruction en Israël (Laetitia Bucaille). Cependant, Bruno Etienne nous rappelle que les victimes les plus nombreuses du kamikazat sont les Musulmans, pensons à la dérive jihadiste meurtrière des "déshérités » de Casablanca ${ }^{4}$, aux attentats du Sinaï, à ceux d'Arabie Saoudite, d'Irak ${ }^{5}$ ou d'Indonésie. Cette violence s'explique par la faillite des gouvernements et des régimes arabes bien entendu, mais également par la perception catastrophique des régimes occidentaux dans le monde musulman. Tout cela complique sérieusement l'analyse de la production/réception de la violence et réintroduit des jeux de discours où, si l'on excepte les combattants suicidaires et leurs commanditaires ainsi que les formes spontanées de satisfaction de la rue arabe, de ressemblances en fausses semblances, le soutien au kamikazat se dessine souvent en creux jusque dans les sociétés cibles (Daniel Hermant).

5 L'archéologie "religieuse » du suicide, son ontogénèse, que nous livre Bruno Etienne, expose l'outillage mental que l'islam met à disposition des kamikazes, outillage d'autant plus facilement réutilisé par ces derniers qu'ils ne sont pas experts en théologie, et en aucune façon soucieux de le devenir. Voilà qui explique l'assimilation fréquente islamisme/terroriste contre laquelle il convient une fois de plus d'être mis en garde. Cependant, cet adossement du kamikazat à un discours idéologique dur n'a rien de spécifique à l'aire musulmane ; on peut le constater ailleurs en élargissant le champ d'observation aux frontières du monde islamique, kurdes (Olivier Grojean), sikhs (Laurent Gayer), au-delà, tamouls (Eleanor Pavey) et, bien sûr, rétrospectivement, japonais. Pour le PKK, Olivier Grojean parle de surintégration au groupe des militants et Laurent Gayer, d'apprentissage de « l'idiome du martyre » pour les Sikhs! Les contenus sont divers et, contre-exemple intéressant, sans dimension religieuse chez les Tamouls, nous dit Eleanor Pavey.

Qu'en est-il de l'Europe ? En première analyse, on ne voit pas très bien ce qu'il pourrait y avoir de commun entre les kamikazes, anciens ou modernes, et une Europe, société cible, qui les voue aux gémonies; et pourtant les choses sont plus complexes. L'article de François Lagrange sur les "combattants de la mort certaine ", nous montre qu'il existe chez nous une gradation d'attitudes entre la mort librement acceptée, voire anticipée, et la mort choisie ou même recherchée, autrement dit que l'analyse ne doit pas être faite en termes binaires qui opposeraient le mode d'action "suicidaire » c'est-à-dire très risqué - au " suicide » qui supprime l'idée même de risque, sauf à aller "à l'encontre d'une véritable compréhension des phénomènes de violence contre soi ", comme le souligne justement Olivier Grojean. Cette hypothèse de continuité entre modes d'action que confirme l'examen historique auquel se livre Louis-Jean Duclos, a une forte valeur heuristique car elle court-circuite la polémique sur la légitimité et 
constitue, bien sûr, un des axes de notre numéro. François Lagrange souligne par ailleurs qu'il existe "un modèle de sacrifice de soi de forte intensité » lié a une élite militaire professionnalisée, mais qu'il a été marginalisé par l'évolution de la guerre, au profit d'une thématique plus modérée du sacrifice de soi (acceptation du risque - même élevé - de mort). On peut d'ailleurs noter que la typologie sur le suicide de Durkheim comporte un "suicide altruiste obligatoire » qui s'ajuste parfaitement au thème que nous étudions. Nulle exceptionnalité européenne donc.

7 Ces détours historiques et méthodologiques montrent que la dimension idéologique, avérée et même tonitruante, ne nous dit rien de fondamental du basculement existentiel ${ }^{6}$ de celui qui va devenir kamikaze, sauf à le réduire à la manipulation ou la mise sous influence, situation qui existe mais qui reste marginale. Concluons qu'il faut chercher l'impulsion suicidaire ailleurs, par exemple, nous suggère Louis-Jean Duclos, dans les bases sociales (le terme est pris ici dans un sens large : forces armées, groupes clandestins révolutionnaires, etc.) constituées précisément en vue d'atteindre un objectif politique par la violence. De ce décentrement de l'analyse, Louis-Jean Duclos, dans sa contribution, tire toutes les conséquences à travers la métaphore juridique du "combat suicidaire " décrit comme un "quasi-contrat» passé entre partenaires. Il nous met en garde contre les chatoyances un peu vaines des discours, en substituant à l'idéologie un marché - souvent de dupes pour le suicidant - qui replace au premier rang la question du but et de l'efficacité stratégique. Or, cette perspective n'est pas souvent envisagée, l'impact considérable du 11 septembre tenant lieu de réponse. C'est pour cela que nous la rappelons dans notre titre " de la mort volontaire combattante", et qu'à côté des articles généraux, nous avons souhaité présenter quelques études de cas qui nous permettent de cerner de plus près cette réalité ${ }^{7}$. Si Eleanor Pavey conclut avec des nuances à l'efficacité stratégique ponctuelle de la méthode, c'est grâce à la "valeur ajoutée " dont les attentats sont affectés en raison de leur exceptionnelle théâtralité. Mais cette règle générale possède une réciproque: leur multiplication correspond à une banalisation, et a donc un rendement décroissant, comme on peut le vérifier au Sri Lanka, en Irak ou en Afghanistan. Laurent Gayer, lui, parle du caractère «a-stratégique» de ces actions et voit dans l'irrésistible désir de mort de nombreux combattants khalistanis une raison de la victoire de l'armée indienne !

Et à l'échelle mondiale ? Un impact considérable bien sûr, mais - en termes politiques ou géopolitiques - des résultats directs dérisoires ou contre-productifs qui évoquent plus la survenance de « coups » qu'une stratégie cohérente.

9 Reste la dimension sociologique. Nos auteurs détaillent les motifs quelquefois minuscules de l'engagement, les profils de la trajectoire: ludique, opportuniste, puisque d'une certaine façon il est possible de faire carrière. Au Sri Lanka, être choisi par l'organisation (LTTE) pour entrer dans les «Tigres noirs », c'est-à-dire l'élite des kamikazes, est un couronnement nous dit Eleanor Pavey. Mais on peut aussi échouer pour incapacité, comme le montre le parcours de Zacarias Moussaoui, pourtant très motivé! Martyropathe ${ }^{8}$ enfin, remarquent nos auteurs en soulignant la forte dimension morbide du phénomène et l'espèce d'injonction contradictoire qui fait passer la victoire par la mort. Voici donc le kamikaze enchainé à son sort, et à l'organisation. Mais cette dépendance est à double sens, car accepter de mourir scelle l'identité du mouvement ${ }^{9}$, transforme les " martyrs volontaires ", en icônes référents de rébellions sacralisées ${ }^{10}$, suscite des attentes bien sûr, mais crée également des obligations. Le compromis ne faisant en aucune façon partie de la panoplie du martyr, 
les dirigeants ne doivent pas décevoir, sauf à risquer d'être désavoués ${ }^{11}$, et à voir le recrutement se tarir, ils sont poussés à l'escalade. Retour au politique donc.

Finalement, entre nos protagonistes il n'y a pas une relation hiérarchique - celle qu'on postule implicitement quand on cherche partout la main d'Al-Qaïda - mais une relation complexe fonctionnant dans les deux sens. Aussi nous n'avons pas, dans notre enquête, cherché à faire apparaitre un modèle général qui n'existe pas, mais plus modestement, selon les conflits, tenté de décrire des configurations paradoxales dans leur fonctionnement car projet politique et dimension sociologique y sont si étroitement intriqués qu'il faut les analyser ensemble, les mettre sous tension en quelque sorte. Le chantier est ouvert, il doit être poursuivi.

Alors que conclure provisoirement? Le politiste serait tenté de dire «qu'il n'y a pas d'avenir significatif pour le suicide de combat et l'attentat suicide en particulier " (Louis-Jean Duclos), mais, à l'inverse, le sociologue constate que l'attractivité des organisations est fondée sur la radicalité des attitudes plus que sur leur efficacité ! Et ce n'est pas le plus rassurant !

\section{NOTES}

1.. Légende de l'illustration de couverture, La Bible, Juges, chapitre 16.30.

2.. Nous nous séparons sur ce point de Robert A. Pape, «The strategic logic of suicide terrorism ", American Political Science Review, vol 97, n³, août 2003.

3.. " "Kamikaze tokubetsu kogekitai" : forces d'attaque spéciales du vent des dieux [...], couramment nommées à l'étranger kamikaze. Nom donné à divers corps de la marine et de l'armée qui se formèrent en vue d'attaques-suicides aux cours des dix derniers mois de la guerre du pacifique. La première attaque eut lieu le 25 octobre 1944. En tout 2198 pilotes se sacrifièrent; 34 navires américains furent coulés et 288 endommagés. », Pinguet M., La Mort volontaire au Japon, Paris, Gallimard, 1984, p. 354.

4.. Qui n'est pas traité dans ce numéro mais qui fait l'objet d'un inédit : Hassan Nour, Sociologie politique du combat suicidaire au Maroc, 2006.

5.. Voir Thomas Hegghammer, Saudis in Iraq: Patterns of Radicalisation and Recruitment, inédit. Traduction française à paraître dans Cultures \& Conflits.

6.. Sur ce point, on peut sans doute regretter qu'au-delà de l'essai de Bruno Etienne sur la « Thanatocratie islamique », la dimension pathologique d'un « kamikazat » qui désinhibe puis exhibe la pulsion de mort n'aie pas été explorée plus avant.

7.. Bien entendu, nous ne visons pas à l'exhaustivité. Il y aurait eu beaucoup à écrire à propos de la guerre de Tchétchénie par exemple, ainsi que sur l'Irak, grand absent de ce numéro où le kamikazat n'est d'ailleurs qu'un modus operandi secondaire.

8.. Nous renvoyons aux trois idéaux types de l'insurgé religieux identifiées par F. Khosrokhavar : ludique, opportuniste, martyropathe, voir : « Le modèle Bassidji », Cultures \& Conflits n²9-30, 1998, pp. 59-118. Laurent Gayer souligne que ce modèle s'applique très bien au Khalistan. 
9.. Cette identité peut prendre une forme très moderne comme dans le cas des « cyberKhalistanis ».

10.. «Te martyrium candidatus laudat exercitus » (« l'éclatante armée des martyrs célèbrent vos louanges »), Te Deum, chant liturgique catholique de rite latin.

11.. L'action suicidaire est aussi un moyen de contester la direction de son propre camp, nous dit Olivier Grosjean.

INDEX

Mots-clés : kamikaze (missions suicide), Stratégie 